\title{
Centri e periferie. Forme e modelli attraverso la storia
}

Storicamente, 2 (2006).

ISSN: 1825-411X. DOI: 10.12977/stor534

Nate all'interno della riflessione sulla nascita dello Stato moderno e debitrici in larga misura di una concezione eurocentrica, che assegnava al vecchio continente e all'Occidente il compito di definire i modelli di riferimento attraverso i quali misurare e comprendere gli 'altri mondi', le categorie interpretative articolate sull'asse spaziale centro-periferia hanno costituito un fondamentale strumento per lo sviluppo delle conoscenze nel campo della storia e delle scienze sociali. Questa capacità euristica del concetto resta sostanzialmente intatta anche oggi, sebbene lo schema binario del rapporto centro periferia sia stato sottoposto, in diversi ambiti disciplinari, a critiche e revisioni che ne hanno evidenziato nuove angolature analitiche e depotenziato la rigida assialità dello schema spaziale. Le "periferie" sono entrate in gioco come protagoniste di una relazione di reciproca influenza con $i$ "centri" e altre categorie sono intervenute a designare la specificità di questi rapporti, man mano che da un mondo di Stati e nazioni si è delineato lo scenario del nuovo mondo globale.

Il dossier intende innanzitutto ripercorrere la complessità di questo schema concettuale e teorico con una particolare attenzione alle costruzioni culturali e ideologiche che hanno intessuto la formazione dello Stato moderno e degli Stati nazionali, attraverso le quali si sono rafforzati o 'inventati' visioni e appartenenze identitarie proiettate sulle periferie. Ma centro-periferia è un modello storiografico che è stato applicato anche a periodi anteriori, come chiave di lettura dei rapporti tra imperi e province, tra città e territori. Da qui la necessità che il dossier assuma un prospettiva di lungo periodo che metta 
in relazione la polis greca all'impero romano, i comuni al Sacro Romano Impero, fino alla nascita degli Stati moderni.

La crisi del modello binario, a cui prima si faceva riferimento, sarà alla base di quei contributi - che via via si raccoglieranno nel dossier - che andranno evidentemente oltre la dimensione nazionale, regionale o locale dello Stato per superare l' immaginario storiografico tendenzialmente eurocentrico e chiamare in causa approcci disciplinari differenti, capaci di mettere in luce le reciprocità tra i due poli del modello in questione. In questo contesto l'evocazione del "sistema mondo" definito e proposto da Wallerstein è quasi d'obbligo, nel duplice senso di ambito nel quale collocare e assumere concettualmente "il primato europeo" che segna tutto il tragitto della modernità, e di osservatorio concettuale nel quale cogliere il valore e il peso della compresenza delle differenze e delle alterità più assolute.

In tale ottica il dossier tenterà di esplorare non solo i processi e le modalità attraverso cui il "centro", nell'atto di conferire significato e senso a mondi sconosciuti - ma anche a mondi pur noti ma sempre «altri» come l'Oriente -, costruisce immagini e narrazioni destinate a riflettersi sulla sua stessa cultura e autorappresentazione, ma anche i processi attraverso i quali "le periferie" hanno adottato i modelli del centro, magari finendo per sottoporli a una critica radicale.

In questo quadro di insieme, privilegeremo cinque filoni principali.

1. II primo riguarda lo "Stato moderno", laddove centro e periferia hanno rappresentato i poli contrapposti della 'modernità' e della 'resistenza' in quei processi che la storiografia otto-novecentesca ha visto condurre in modo unilineare - attraverso la fase assolutistica e poi quella rivoluzionaria - alla centralizzazione politica, alla razionalizzazione burocratica e militare, all'unificazione culturale e linguistica culminante nello Stato-nazione. Gli studi degli ultimi tre decenni, soprattutto degli anni Novanta in coincidenza con l'accelerarsi della creazione di un'entità statale europea sopranazionale, hanno contribuito a rivedere tale rapporto tra centro e periferia. Da una 
parte, su scala continentale e macro-storica si è messo in evidenza l'anacronismo insito in quell'idea di "Stato moderno" e di "Stato-nazione": gli Stati in età moderna - la Spagna degli Asburgo, le isole britanniche dai Tudor agli Hannover, la stessa Francia di Luigi XIV - furono piuttosto entità composite, dove differenti unità culturali e politiche furono unite sotto un unico sovrano; una situazione in cui piuttosto che imporre politiche dal "centro", la concezione ordinaria di governo prevedeva il rispetto delle istituzioni e delle culture giuridiche e politiche locali e le sue pratiche consistevano in una complessa opera di negoziazione della corte con le élites e gli istituti rappresentativi provinciali. Dall'altra l'idea di una complessa interazione tra centro e periferia è stata studiata su scala regionale e microstorica, ponendo in luce la molteplicità di "centri" e "periferie" individuabili nelle realtà politiche e istituzionali di età moderna. In questa prospettiva, la storia europea viene a caratterizzarsi come una continua tensione: la "modernità" dello Stato europeo scaturisce da questa tensione tra le istanze di accentramento e le tendenze alla dispersione del potere, dalla capacità di recepire e adattare le proprie istituzioni al pluralismo giuridico e istituzionale che gli preesiste.

2. Un altro campo di interventi riguarderà l'evoluzione di questa dialettica centro/periferia costitutiva dello Stato moderno in età contemporanea. L'età rivoluzionaria e quella dell'Impero napoleonico sono state assunte a momento fondativo di un decisivo cambiamento nei rapporti di potere allinterno dei confini statali. Di tale cambiamento si sono studiati gli effetti: le dinamiche e le caratteristiche del confronto tra le due parti, oscillante tra i poli ideali della perfetta integrazione e della totale opposizione.

Da una parte, avviene il definitivo consolidamento dello Stato, che arriva ad estendere la sua giurisdizione in ogni ambito della vita pubblica, sottraendo competenze e prerogative ai ceti sociali, alla Chiesa, alle comunità. II rapporto centro-periferia ha richiesto da questo momento letture analitiche delle dinamiche con cui si è attuato l'adattamento della gerarchia piramidale al territorio, imponendosi o incontrandosi con esigenze e resistenze 
particolari. Adottando il punto di vista dello Stato, le categorie di indagine sullo "spazio nazionale" hanno mostrato la tendenza a identificare il centro con il paese, le istituzioni centrali con la vita politica nazionale, e la stessa capitale con il luogo di formazione e irraggiamento della cultura nazionale 'ufficiale'.

Dall'altra parte, l'avanzata del centro ha imposto alle periferie una rielaborazione del proprio patrimonio identitario in senso funzionale ai ripetuti tentativi di emancipazione. Nasce da questa forzata dialettica, come ha sottolineato la storiografia francese, la categoria di provincia come entità storico-culturale autonoma, non riducibile alla subalternità di cui si connota il termine periferia. In altri contesti nazionali, l'unità di riferimento è la città / comune o la regione. Anche il concetto di patrimonio, inteso come corpus di testimonianze naturali, monumentarie, simboliche, della individualità / alterità della periferia, provincia regione o comune, rispetto alla capitale; così come quello di territorio, come unità fisico-antropologica a se stante, devono molto a questo processo di rielaborazione, e alla affermazione di scale di indagine alternative a quella statale. Riemerse come autonomo oggetto di studio già con la scuola di Labrousse, le periferie hanno infine acquisito il 'rango' di tasselli fondamentali per lo studio delle storie, delle identità, delle culture nazionali contemporanee.

3. Altri contributi metteranno alla prova le capacità euristiche del modello centro-periferia nel campo degli studi delle religioni e delle chiese. Ci interessa indagare come le religioni sviluppino un particolare immaginario su centro e periferia, legato al rapporto con determinati luoghi di culto e devozione (sia dal punto di vista geografico e istituzionale, sia dal punto di vista meramente culturale), e come religioni delle periferie, come quelle del Medio Oriente antico, abbiano potuto imporsi al centro istituzionale dell'impero fino a divenire dominanti.

I saggi che andremo a pubblicare analizzeranno inoltre le modalità con cui le diverse religioni - in primo luogo ebraismo, cristianesimo e islam - 
organizzano al loro interno il rapporto tra centri e periferie sotto il profilo istituzionale. Mentre il cristianesimo ha risolto tale rapporto in una continua situazione di crisi e adattamento, in un'oscillazione ricorrente tra l'idea di un centro universale e una istanza locale, periferica, che si può autonomizzare dopo momenti di rottura, l'ebraismo, privo di un centro politico e territoriale per almeno due millenni, sopravvive con una forma istituzionale debole in una diaspora senza centro, sviluppando forme peculiari di riflessione e sacralizzazione nei confronti del luogo simbolico di Gerusalemme. L'islam, a sua volta, pur sperimentando un'espansione politica simile a quella del cristianesimo, si è strutturato sotto il profilo religioso e istituzionale con modalità molto differenti proprio in termini di rapporto centro/periferia.

Si terranno per altro in considerazione anche le forme di sacralizzazione del territorio e dello spazio: ciò aiuta a comprendere, in particolare, le strategie attraverso cui cristianesimo e islam, in quanto religioni con vocazione universale, hanno "colonizzato" spazi lontani, sia dal punto di vista culturale che geografico, imponendosi su altre forme di religiosità. Dal punto di vista culturale è utile analizzare come le religioni organizzino e traducano in termini religiosi lo spazio, attraverso l'invenzione di culti locali, di pellegrinaggi e percorsi sacri che segnano profondamente le città e il territorio in generale.

Infine, soprattutto per quanto concerne l'età moderna, verranno considerate le modalità con cui i cristianesimi di diversa confessione si sono confrontati con altre culture nel momento in cui l'Europa cristiana andò espandendosi al di fuori dei suoi confini storici. In questo senso è utile capire come, ad esempio, la Chiesa di Roma si sia organizzata sui territori delle colonie spagnole o ancora come abbia elaborato una strategia culturale di evangelizzazione in Oriente.

4. Un'altra area di intervento del dossier riguarda la sfida lanciata dagli studi postcoloniali all'etnocentrismo delle narrazioni storiografiche occidentali ed il rapporto tra questi nuovi studi di impianto culturale e le analisi storico- 
economiche della World History. Evento periodizzante nel panorama di questi studi è stata la pubblicazione nel 1979 del volume di Said, Orientalismo, perché ha attirato l'attenzione sulla crucialità delle rappresentazioni dell'Oriente nell'autorappresentazione identitaria della modernità occidentale. Da questi presupposti critici si sono sviluppati diversi filoni intellettuali che dagli ambiti dell'estetica e della critica letteraria hanno mosso alla filosofia (in particolare nel confronto con il pensiero decostruzionista) e stanno interessando la ricerca storica. Tra gli indirizzi storiografici più promettenti, che rovesciano l'impianto analitico di un centro che «guida» la periferia, vanno certamente annoverati gli studi del collettivo indiano Subaltern Studies, animato all'inizio degli anni '80 da Ranjit Guha. Questi studi hanno insistito sulla centralità della azione delle classi subalterne nella costruzione di una specifica modernità indiana, criticando al contempo la storiografia colonialista e quella di impianto nazionalistico, che ha acquisito gli stessi paradigmi della storiografia occidentale, spostando semplicemente la figura sociale guida del processo di modernizzazione, dall' élite coloniale all'élite nazionale. Questa attenzione alla agency delle classi subalterne, che non a caso trova in Gramsci un fondamentale riferimento teorico, viene coniugata attraverso nuovi modi di rappresentare lo spazio, che hanno come denominatore comune la critica dell'assunzione della Nazione e dello Stato come pilastri della costruzione delle narrazioni storiche. Da qui hanno mosso studiosi delle diaspore e dei sistemi di relazioni multiple, i quali hanno messo definitivamente in crisi una semplice relazione assiale tra centro e periferia: gli studi sull'oceano Indiano prima e dopo l'ingresso di portoghesi, olandesi ed inglesi, così come l'analisi delle triangolazioni atlantiche alla base dei movimenti demografici, politici, economici che hanno disegnato il volto dell'epoca moderna. Attraverso la critica postcoloniale, e gli sviluppi che essa ha generato in ambito storiografico, diviene perciò possibile coniugare sofisticate analisi di storia della cultura con metodologie più «dure» e quantitative che analizzano su scala globale temi classici della storia economica quali quello della nascita e diffusione del capitalismo commerciale e industriale. 
5. Un ultimo filone di indagine perseguito da alcuni saggi riguarda gli studi geografici, all'interno dei quali il modello centro/periferia ha costituito una metafora imprescindibile nel loro quadro concettuale e epistemologico. Trovando terreno fertile in una geografia sviluppatasi tra le due guerre, nella quale il discorso geografico ribadisce il sopravvento - anche negli approcci quantitativi - sul fiscalismo cartografico, tale metafora ha avuto la pretesa di proporre, in uno spazio al contempo politico, economico e culturale, modelli interpretativi basati sull'ordine della geometria euclidea. II declino dell'uso del modello centro/periferia così concepito si è fatto particolarmente sensibile nel corso dell'ultimo ventennio. Esso ha avuto origine da una parte nella critica ad una visione "semplicistica ed ingannevole" delle dinamiche spaziali che non tengono conto delle nuove compressioni spazio/temporali, dall'altra in un "ritorno" ad una forma discorsiva basata sull'analisi delle espressioni e delle intenzioni del soggetto, oltre che delle identità collettive. Entità, queste, per le quali la dialettica centro/periferia si trasforma in relazione con una alterità "incommensurabile" - su scale diverse - in un contesto spaziale disomogeneo, discontinuo, anisotropo nel quale le reti diventano fattori di coesione e al contempo generatori di complessità. II risultato di questa tendenza è una crisi delle tradizionali categorie spaziotemporali che, alimentata dai fenomeni di globalizzazione, agisce sconvolgendo gli assetti geografici su diverse scale: dalle configurazioni urbane alle trasformazioni del contesto globale. In tale panorama il discorso geografico non può prescindere dai forti, continui cambiamenti nella percezione e nella rappresentazione del mondo. Cambiamenti, questi, veicolati anche dal recente sviluppo di strumenti analitici e comunicativi, quali i sistemi informativi geografici, diffusi largamente, anche nella pratica del "quotidiano", attraverso Internet.

\section{Alcune letture a proposito di centri e periferie}




\section{Per l'età moderna e contemporanea}

M. Agulhon, Le centre et la périphérie, in $\mathrm{P}$. Nora (ed.), Les lieux de mémoire , Paris, Quarto-Gallimard, 1997, II, 2889-2906.

P. Anderson, Lo Stato assoluto, Milano, Mondadori, 1980.

G. Arrighi, Il lungo 20. secolo. Denaro, potere e le origini del nostro tempo, Milano, Il saggiatore, 1996.

B. Baldi, Stato e territorio. Federalismo e decentramento nelle democrazie contemporanee, Roma-Bari, Laterza, 2002.

G. Chittolini, A. Molho, P. Schiera (eds.), Origini dello Stato: processi di formazione statale in Italia fra Medioevo ed età moderna, Bologna, II Mulino, 1994.

Ch. Charle, D. Roche (eds.), Capitales culturelles, capitales symboliques: Paris et les expériences européennes, Paris, Publications de la Sorbonne, 2002.

Ch. Charle (ed.), Capitales culturelles et rayonnement symbolique, Paris, Editions Rue d'Ulm, 2004.

L. Colley, Prigionieri. L'Inghilterra, I'Impero e il mondo. 1600-1850, Torino, Einaudi, 2004.

J.H. Elliott, An Europe of Composite Monarchies, «Past and Present», 137 (1992), pp. 48-71.

O. Gaspari, P. Dogliani (eds.), L'Europa dei Comuni: origini e sviluppo del sistema comunale europeo dalla fine dell'Ottocento al secondo dopoguerra, Roma, Donzelli, 2003.

K. Pomeranz, La grande divergenza: la Cina, l'Europa e la nascita dell'economia mondiale moderna, Bologna, II Mulino, 2004.

A. Romano, Il modello costituzionale inglese e la sua ricezione nell'area del Mediterraneo tra la fine del '700 e la prima metà dell' '800 
, Milano, Giuffrè, 1998.

P. Rosanvallon, Le modèle politique français: la société civile contre le jacobinisme de 1789 à nos jours, Paris, Editions du Seuil, 2004.

E. Rotelli, P. Schiera, Lo Stato moderno, Bologna, II Mulino, 1974-1976, 3 voll.

P. Schiera (ed.), Le autonomie e l'Europa. Profili storici e comparati, Bologna, il Mulino, 1993.

C. Tilly (ed.), La formazione degli stati nazionali nell'Europa occidentale, Bologna, il Mulino, 1984.

\section{Per il mondo antico:}

P. Bilde, T. Engberg-Pedersen, L. Hennestad, J. Zahle, K. Randsborg (eds.), Centre and Periphery in the Hellenistic World, Studies in Hellenistic Civilization 4, Aarhus, 1993.

P. Horden, Nicholas Purcell, The Corrupting Sea. A Study of Mediterranean History, Blackwell, 2000.

M. Rowlands, M. Larsen, K. Kristiansen (eds.), Centre and Periphery in the Ancient World, Cambridge University Press, Cambridge, 1987.

G. Woolf, C. Edwards, Rome the Cosmopolis, Cambridge University Press, Cambridge, 2003.

\section{Negli studi post-coloniali:}

D. Chakrabarty, Provincializzare l'Europa, Roma, Meltemi, 2004.

P. Gilroy, The Black Atlantic. L'identità nera tra identità e doppia coscienza, Roma, Meltemi, 2003.

R. Guha, La storia ai limiti della storia del mondo, Firenze, Sansoni, 2003.

R. Guha, G. Spivak, Subaltern studies. Modernità e (post)colonialismo, 
Verona, Ombre Corte, 2002.

P. Linebaugh, M. Rediker, I ribelli dell'Atlantico. La storia perduta di un'utopia libertaria, Milano, Feltrinelli, 2004.

S.W. Mintz, Storia dello zucchero: tra politica e cultura, Torino, Einaudi, 1990.

E. Said, Orientalismo. L'immagine europea dell'oriente, Milano, Feltrinelli, 2001.

E. Said, Cultura e imperialismo: letteratura e consenso nel progetto coloniale dell'Occidente, Roma, Gamberetti, 1998.

\section{Nella storia delle religioni:}

G. Filoramo, Che cos'è la religione? Temi, metodi, problemi, Torino, Einaudi, 2004.

J.Z. Smith, Map Is Not Territory: Studies in the History of Religions, Chicago, University of Chicago Press, 1978.

J.Z. Smith, Relating Religion. Essays in the Study of Religion, Chicago, University of Chicago Press, 2004.

\section{Nel campo della geografia:}

M. Dodge, R. Kitchin, Mapping cyberspace, Londra - New York, Routledge, 2001.

J. Gottmann (ed.), Centre and periphery: spatial variation in politics, Londra, Sage, 1980.

D. Harvey, The condition of postmodernity: an enquiry into the origins of cultural change, Cambridge Mass. - Oxford, Blackwell, 1990 (trad. it., La crisi della modernità, Milano, II Saggiatore, 1997).

M. Pacione, Urban geography: a global perspective, Londra - New York, 
Routledge, 2001.

P. Petsimeris (ed.), Le trasformazioni sociali dello spazio urbano, Bologna, Patron, 1991.

C. Raffestin, Territorialità e paradigma centro-periferia: la Svizzera e la Padania, Milano, Unicopli, 1978. 